\title{
MIR144 Pre-miRNA
}

National Cancer Institute

\section{Source}

National Cancer Institute. MIR144 Pre-miRNA. NCI Thesaurus. Code C82774.

MIR144 is an oligoribonucleotide that is encoded by the human MIR144 gene and has a role in the regulation of gene expression. 